\title{
Programmable RNA detection with a fluorescent RNA aptamer using optimized three-way junction formation
}

\author{
YUICHI FURUHATA, ${ }^{1,6}$ MIZUKI KOBAYASHI, ${ }^{2,6}$ RYO MARUYAMA, ${ }^{3}$ YUSUKE SATO, ${ }^{4}$ KURUMI MAKINO, ${ }^{2}$ \\ TATSUO MICHIUE, ${ }^{3}$ HIROHARU YUI, ${ }^{2}$ SEIICHI NISHIZAWA, ${ }^{4}$ and KEITARO YOSHIMOTO ${ }^{3,5}$ \\ ${ }^{1}$ Biomedical Research Institute, National Institute of Advanced Industrial Science and Technology (AIST), Tsukuba 305-8566, Japan \\ ${ }^{2}$ Department of Chemistry, Faculty of Science, Tokyo University of Science, Tokyo 162-8601, Japan \\ ${ }^{3}$ Department of Life Sciences, Graduate School of Arts and Sciences, The University of Tokyo, Tokyo 153-8902, Japan \\ ${ }^{4}$ Department of Chemistry, Graduate School of Science, Tohoku University, Sendai 980-8578, Japan \\ ${ }^{5}$ JST, PRESTO, The University of Tokyo, Tokyo 153-8902, Japan
}

\begin{abstract}
RNAs play essential roles in various cellular processes and can be used as biomarkers. Hence, it is important to detect endogenous RNA for understanding diverse cellular functions and diagnosing diseases. To construct a low-cost and easy-touse RNA detection probe, a chemically unmodified RNA aptamer that binds to a pro-fluorophore to increase its fluorescence is desirable. Here, we focused on Broccoli, a superior variant of Spinach, which is a well-known fluorescent RNA aptamer that binds to DFHBI-1T and emits green fluorescence. We experimentally characterized Broccoli and predicted that it forms a G-quadruplex-based DFHBI-1T recognition region sandwiched between two stems. Based on this, we designed a Broccoli-based RNA detection probe (BRD probe) composed of a sequence of destabilized Broccoli fused with complementary sequences against target RNA. The resulting probe with its target RNA formed a stable three-way junction, named the MT2 three-way junction, which contributed to efficient refolding of the Broccoli structure and allowed for programmable RNA detection with high signal-to-noise ratio and sensitivity. Interestingly, the MT2 three-way junction also could be applied to probe construction of a truncated form of Spinach (Baby Spinach). The BRD and Baby Spinachbased RNA detection probes (BSRD probe) exhibited up to 48- and 140-fold fluorescence enhancements in the presence of their target RNAs and detected small amounts of target RNA that were as low as 160 and 5 nM, respectively. Thus, we experimentally characterized the higher order structure of Broccoli and developed structure-switching aptamer probes for highly sensitive, programmable, RNA detection using an MT2 three-way junction.
\end{abstract}

Keywords: fluorescent RNA; Broccoli; Baby Spinach; RNA sensor; three-way junction

\section{INTRODUCTION}

RNAs play important roles in various cellular processes. In protein synthesis, mRNA is the translation template, whereas tRNAs and rRNAs transport and catalyze the polymerization of amino acids, respectively (Alberts et al. 2008). In living cells, the dynamic expressions of these RNAs directly affect diverse biological phenomena, such as cell division, cellular differentiation, and circadian rhythm (Chiu et al. 2004; Martin and Ephrussi 2009). To assess gene function and understand diverse cellular mechanisms, it is important to detect these RNAs. Furthermore, since many disease-related cells secrete specific RNAs, RNA detection methods

\footnotetext{
${ }^{6}$ These authors contributed equally to this work.

Corresponding authors: ckeitaro@mail.ecc.u-tokyo.ac.jp, y-furuhata@aist.go.jp

Article is online at http://www.rnajournal.org/cgi/doi/10.1261/rna. 069062.118
}

have attracted major attention for disease diagnosis and medical treatment (Li et al. 2014; Xia et al. 2017).

For in vitro RNA detection, various methods have been proposed. Among these methods, reverse transcriptionpolymerase chain reaction (RT-PCR) and the use of molecular beacon (MB) probes are representative of indirect and direct detection methods, respectively. In RT-PCR, cDNA can be synthesized from disease-specific RNAs via a reverse transcription reaction followed by amplification and detection using quantitative PCR (Higuchi et al. 1992; Freeman et al. 1999). Since this simple but effective method enables for highly sensitive detection of target RNAs, it has been used for many years. However, these steps are

(c) 2019 Furuhata et al. This article is distributed exclusively by the RNA Society for the first 12 months after the full-issue publication date (see http://rnajournal.cshlp.org/site/misc/terms.xhtml). After 12 months, it is available under a Creative Commons License (Attribution-NonCommercial 4.0 International), as described at http:// creativecommons.org/licenses/by-nc/4.0/. 
time-consuming and require costly reagents. Moreover, RT-PCR cannot detect endogenous RNA directly. Several groups reported direct detection of endogenous RNAs using an MB probe (Tyagi and Kramer 1996; Mao et al. 2009; Yeh et al. 2010; Xia et al. 2017). MB probes form a stemloop structure with a fluorophore at one end and a quencher at the other end in the absence of the target RNA. When the MB probe hybridizes to the target RNA, the quencher is separated from the fluorophore, allowing for emission of a fluorescent signal upon excitation. Although MB probes enable for direct detection of endogenous RNAs, they are difficult to design and require costly reagents for the chemical modification step. Moreover, MB probes require microinjection to introduce them into target cells and this is also a strong limitation.

For the construction of low-cost and easy-to-use endogenous RNA detection probes, a chemically unmodified RNA aptamer that binds to a pro-fluorophore to increase its fluorescence is desirable. Recently, Sato et al. (2015) reported an endogenous RNA imaging system using a blackhole-quencher (BHQ)-binding RNA aptamer, whereas Ong et al. (2017) reported another endogenous RNA imaging system using a variant of 3,5-difluoro-4-hydroxybenzylidene imidazolinone (DFHBI)-binding RNA aptamer, named Spinach. RNA probes in both systems consist of a destabilized RNA aptamer sequence and recognition sequences that are complementary to the target RNA. These probes are stabilized only upon hybridizing to the target RNA and form a complex with a partner pro-fluorophore, resulting in fluorescence enhancement. However, these systems have poor signal-to-noise ratios and difficulty detecting low-abundance RNAs.

In this study, we produced a highly sensitive RNA detection probe, named the Broccoli-based RNA detection probe (BRD probe) and the Baby Spinach-based RNA detection probe (BSRD probe). Broccoli is one of the brightest variants of Spinach, which binds to the GFP fluorophore-like pro-fluorophore, DFHBI, and its derivative DFHBI-1T, which activates their green fluorescence (Filonov et al. 2014; Autour et al. 2016; Svensen and Jaffrey 2016; Okuda et al. 2017). The construction scheme of the BRD probe is illustrated in Figure 1. An RNA detection probe should dissociate from the pro-fluorophore without its target RNA; thus, a destabilized form of the RNA aptamer is needed. We initially performed structural characterization of the wild-type (WT) Broccoli-DFHBI-1T complex to design its destabilized form. The structure of the WT Broccoli-DFHBI-1T complex was predicted to have a G-quadruplex-based DFHBI-1T recognition region sandwiched between two stems, named P1 stem and P2 stem [Fig. 1 (i)]. Based on the predicted structure, the P1 stem was truncated to destabilize the Broccoli-DFHBI-1T complex, as shown in Figure 1 (ii). Then, we constructed a probe that has a destabilized Broccoli sequence fused to target recognition sequences through mononucleotide bridges [shown in blue and yellow in Fig. 1 (iii)]. Since the initial Broccoli probe could not refold into the Broccoli structure or stimulate DFHBI-1T fluorescence, we introduced mutations at the $\mathrm{P} 1$ stem to improve the refolding efficiency. As shown in Figure 1 (iv), the optimized Broccoli probe with the target RNA formed a stable three-way junction and could bind and stimulate enhancement of the fluorescent signal of DFHBI-1T. We named this the BRD probe. Interestingly, the optimized three-way junction formation could also be applied to probe construction using Baby Spinach, a minimized variant of Spinach. Our design strategy to construct structure-switching aptamers, which is based on the formation of a three-way junction using the mutant stem sequence, provided two programmable RNA detection probes with high sensitivity and specificity.

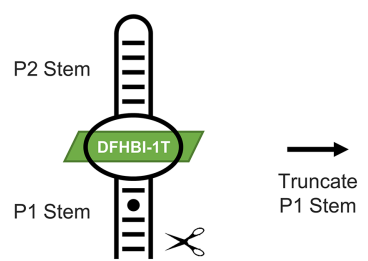

(i) Structurally Characterized Wild-type Broccoli

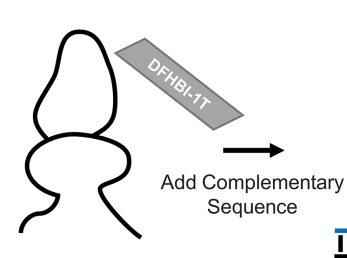

(ii) Destabilized Broccoli

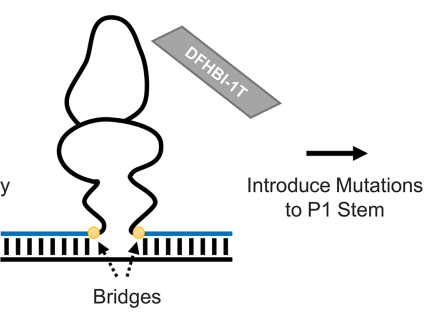

(iii) Initial Broccoli Probe with Target RNA

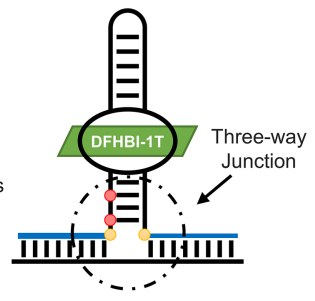

(iv) Optimized Broccoli Probe with Target RNA

FIGURE 1. Schematic overview for the construction of the Broccoli-based RNA detection probe. (i) The predicted structure of the BroccoliDFHBI-1T complex. Structural characterization was performed using sequence alignment, circular dichroism, and UV melting measurements. Broccoli was predicted to have two stems (P1 and P2). Refer to Figure 2. (ii) The destabilized form of Broccoli. The P1 stem was truncated to destabilize Broccoli and quench the fluorescence of DFHBI-1T. (iii) The initial Broccoli probe consisted of a destabilized Broccoli, mononucleotide bridges (yellow), and the target recognition sequences complementary to the target RNA (blue). This probe could not enhance the fluorescence of DFHBI-1T even upon hybridizing with its target RNA. (iv) The optimized Broccoli probe, which carries nucleobase mutations (red) at the P1 stem. The optimized Broccoli probe refolded upon hybridizing with its target RNA and formed a complex with DFHBI-1T, resulting in enhancement of DFHBI-1T fluorescence. This probe-target RNA complex formed a stable three-way junction (dashed circle). 


\section{RESULTS AND DISCUSSION}

\section{Structural characterization of Broccoli-DFHBI-1T complex}

For RNA detection, the RNA probe and DFHBI-1T should not form a complex in the absence of the target RNA. Thus, a destabilized form of the RNA aptamer is necessary. Since the 3D structure of Broccoli has not been elucidated, we initially predicted the 3D structure of Broccoli. The 3D structure prediction of Broccoli was performed based on the previously reported crystal structure of Spinach (Paige et al. 2011; Song et al. 2014; Warner et al. 2014). According to previous studies, Spinach adopts a G-quadruplex structure for DFHBI-1T recognition that is sandwiched between the two stems, P1 and P2 (Huang et al. 2014; Warner et al. 2014). By comparing the primary sequences of Broccoli with a part of Spinach, it was revealed that both sequences were very similar, especially in the DFHBI-1T recognition region, with only two base conversions (Fig. 2A). Although the other parts of Broccoli are quite different from Spinach, we speculated that these sequences formed two stems (P1 and P2 in Fig. 2A) due to their base complementation, suggesting that Broccoli forms a stem-loop structure with the G-quadruplex, similar to Spinach.

To confirm our speculation, the structural characterization of Broccoli was performed using CD and UV melting measurements. $C D$ spectra are often used to check for the presence of G-quadruplex structures in nucleic acids. G-quadruplex-containing RNAs, including Spinach derivatives, show a positive band at $264 \mathrm{~nm}$ (DasGupta et al. 2015). In this study, almost all experiments were performed in HEPES buffer with the exception of the CD experiments, which were performed in Tris buffer to facilitate measurements recorded at wavelengths $<240 \mathrm{~nm}$. The CD spectra of Broccoli were measured, and a truncated Spinach (Baby Spinach) was used as a control sample. As shown in Figure 2B, Broccoli had a positive band at $264 \mathrm{~nm}$ and the spectrum was overall quite similar to that of Baby Spinach. A positive band at $264 \mathrm{~nm}$ was also observed in HEPES buffers (Supplemental Fig. S1). Next, the UV melting measurements were performed and revealed the presence of a stem structure in
Broccoli. As shown in Figure 2C, the absorbance of both Broccoli and Baby Spinach at $260 \mathrm{~nm}$ increased in a temperature-dependent manner, indicating that Broccoli has a double-stranded structure like stem. Moreover, the increase of the absorbance appeared to be in two steps, suggesting that Broccoli has two stem structures. The above results suggest that Broccoli consists of a G-quadruplex and a stem structure, as illustrated in the left panel of Figure 2D. In addition, the predicted 3D structure of Broccoli was constructed by molecular modeling using the PDB structure of Spinach (the right of Fig. 2D). In the initial structure, two nucleobases at 27A and $62 \mathrm{U}$ in the DFHBI-1T recognition region of Spinach were flipped out from the quadruplex core. Interestingly, only these two nucleobases were substituted in the DFHBI-1T recognition region of Broccoli, 
suggesting that these mutations have little effect on the higher-ordered structure formation of the DFHBI-1T recognition region (Fig. 2D). Consistent with these considerations, a nucleobase at 27A was also pointed out to be tolerant to mutations in Spinach in previous studies (Ketterer et al. 2015; Autour et al. 2016). These results reveal that the structure of Broccoli is very similar to that of Baby Spinach and has a G-quadruplex-based DFHBI-1T recognition region sandwiched between the P1 and P2 stems. Recently, Ageely et al. (2016) also suggested that Broccoli and Baby Spinach are likely to possess very similar structures, including nearly identical G-quadruplex cores. The predicted 3D structure of Broccoli in this study was consistent with their report.

Since the P1 stem is important for regulating the thermodynamic stability and fluorescence activation property of Spinach (Huang et al. 2014), we hypothesized that mutation or truncation of the P1 stem would decrease the thermodynamic stability and fluorescence activation properties of Broccoli. To test this, five truncated forms of Broccoli at the P1 stem were synthesized using in vitro transcription (Supplemental Tables S1, S2). Their fluorescence activation properties with DFHBI-1T were studied and the effects of truncating the number of base pairs were investigated. As shown in Figure 2E, truncation of the P1 stem of Broccoli decreased its fluorescence activation property. Interestingly, a truncated Broccoli possessing a 4-bp stem length dramatically decreased its fluorescence activation property compared to WT Broccoli, which has a 5bp stem length. These results indicate that the 1-bp truncation was effective and could be used to construct the thermodynamically destabilized Broccoli. Therefore, we focused on engineering the truncated form of Broccoli that possesses a 4-bp stem length that was then used to construct an RNA detection probe.

\section{Design of stem structure for BRD probe}

As a prototype of the BRD probe, two 10-nt RNA sequences complementary to target the RNA (5'-ACU ACA GCU GAU CCG CGU ACC AUC CAU UAA CAA GCU ACA GUA CAC CAA AAC A-3') were fused to the $5^{\prime}$ and $3^{\prime}$ ends of the truncated Broccoli possessing the 4-bp stem length through $U$ mononucleotide bridges (Fig. 3A and the left of Fig. 3B). Target recognition sequences and $U$ mononucleotide bridges are shown in blue and yellow, respectively. This construct was named the WT probe. Since the distance between the $5^{\prime}$ and $3^{\prime}$ ends of the truncated Broccoli was estimated to be $17.35 \AA$ based on the 3D structure prediction, we created a 4-nt, single-stranded structure at the center of the target RNA, UUAC, which is highlighted in purple on the left panel in Figure 3B. As shown in Figure 3C, the WT probe did not show any fluorescence enhancement in the presence of the target RNA. Then, we speculated that the terminal $A-U$ bases at the
P1 stem were too inflexible to form hydrogen bonds. Thus, we exchanged the terminal A-U base pair at the P1 stem to a G.U wobble base pair, which has more structural flexibility compared to Watson-Crick base pairs (Varani and McClain 2000). This modified probe was named the mutant 1 (MT1) probe (Fig. 3A and the middle of Fig. 3B). Although the MT1 probe showed a 1.7-fold enhancement of fluorescence intensity in response to binding the target RNA, the fluorescence intensities in the presence and absence of the target RNA were strongly decreased compared to the WT probe (Fig. 3C). The fluorescence intensity of the probe is an important factor for measuring fluorescent signals with high sensitivity and accuracy. The fluorescence intensity of the MT1 probe could be enhanced by improving the thermodynamic stability of the P1 stem. The mismatched A-G pair at the P1 stem was then modified to a C-G base pair, and the modified probe was called MT2 (Fig. 3A and the right of Fig. 3B). As expected, the fluorescence intensity of the MT2 probe in the absence of the target RNA was increased, probably due to the higher thermodynamic stability of the P1 stem compared to that of the MT1 probe (Fig. 3B,C). Moreover, the MT2 probe showed a 2.7-fold enhancement of fluorescence intensity in response to binding the target RNA (Fig. 3C). Based on these results, we accomplished construction of an RNA detection probe based on Broccoli by optimizing the P1 stem structure. While the MT2 probe possesses a thermodynamic stability of its P1 stem and fluorescence intensity in the absence of the target RNA comparable to the WT probe (Fig. 3B,C), their fluorescence enhancement properties against target RNA were quite different (Fig. 3C). Considering these findings, the structural flexibility of the G.U wobble base pair greatly contributed to efficient refolding of the Broccoli structure with a three-way junction and activation of the DFHBI-1T fluorescence. We refer to this structure as the MT2 threeway junction (Fig. 3D).

We investigated the effect of the target recognition sequence length on fluorescence enhancement of the MT2 probe. For this purpose, MT2 probes with 10-nt, 12-nt, and 14-nt target recognition sequences were constructed. As shown in Figure 3E, the MT2 probe with the 14-nt target recognition sequences showed the maximum enhancement, with a 12-fold enhancement of the target RNA-dependent increase in fluorescence intensity. Surprisingly, extension of the target recognition sequence not only increased the fluorescence intensity of the probe complexed with the target RNA, but also decreased the background signal of the probe in the absence of the target RNA (Fig. $3 \mathrm{E})$. Since extension of the target recognition sequences probably increases the structural diversity of the probe RNA in the absence of the target RNA, it might suppress the undesired folding of Broccoli and activation of DFHBI-1T fluorescence. We used the resultant MT2 probe with the 14-nt target recognition sequence in subsequent studies and refer to this construct as the BRD probe. 
A

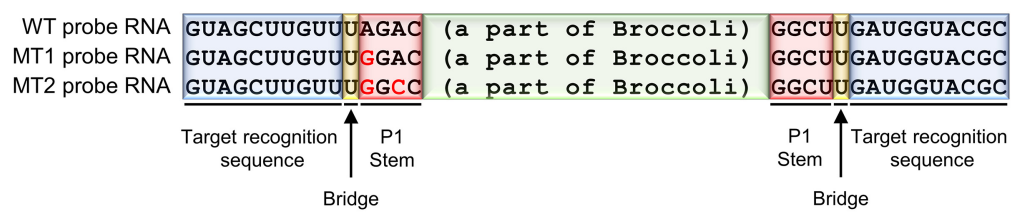

B
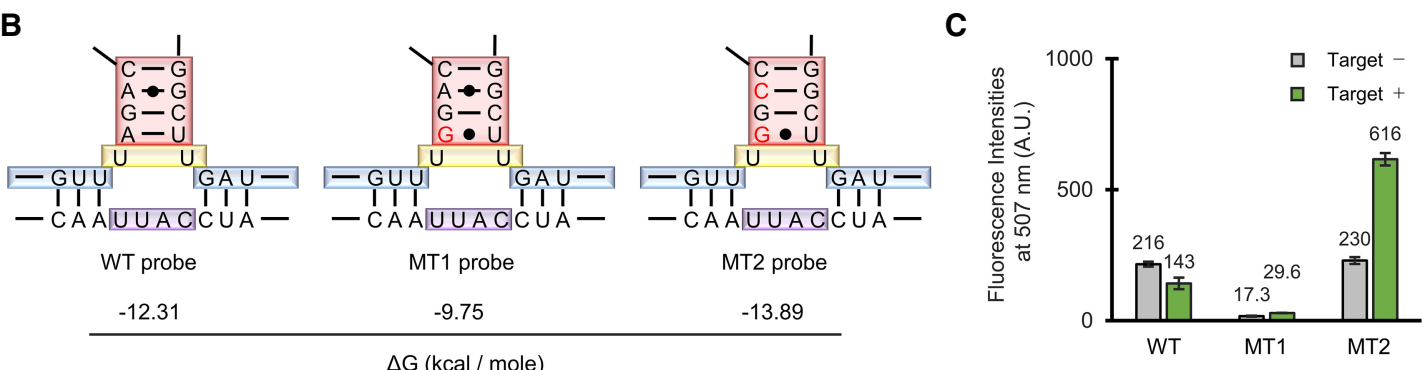

D

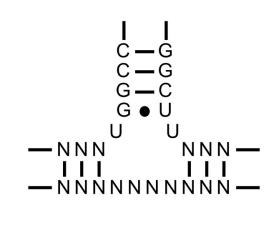

E

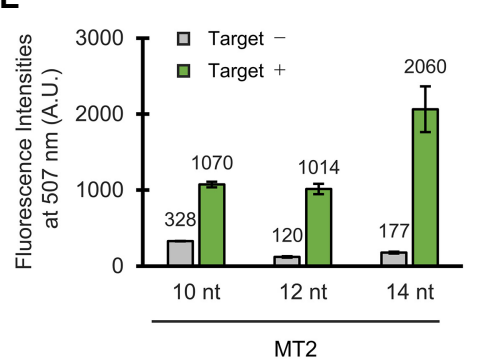

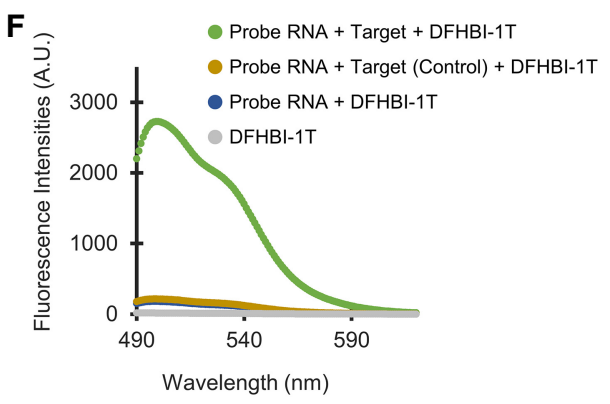

FIGURE 3. Construction and optimization of the BRD probe. (A) Sequence alignment of WT, mutant 1 (MT1), and mutant 2 (MT2) probe RNAs. (B) Secondary structure illustrations of the three-way junctions of WT, MT1, and MT2 probe RNAs. Mutated nucleotides from the WT probe are shown in red. P1 stems, U mononucleotide bridges, target recognition sequences, and 4-nt margins in target RNAs are highlighted in red, yellow, blue, and purple, respectively. Gibbs free energies of the individual P1 stems were calculated using mfold. (C) Fluorescence intensities of Broccolibased probes possessing different three-way junctions for target recognition. The $10 \mu \mathrm{M}$ probe RNA was incubated in the absence (gray) or presence (green) of $10 \mu \mathrm{M} 2 \times$ target RNA with $10 \mu \mathrm{M}$ DFHBI-1T. Values shown are the means \pm SEs of $n=3$. (D) A secondary structure illustration of the MT2 three-way junction. (E) Fluorescence intensities of the MT2 probe possessing different lengths of target recognition sequences for target recognition. The $10 \mu \mathrm{M}$ probe RNA was incubated in the absence (gray) or presence (green) of $20 \mu \mathrm{M} 1 \times \operatorname{target}$ RNA with $10 \mu \mathrm{M}$ DFHBI-1T. Values shown are the means \pm SEs of $n=3$. $(F)$ Fluorescence spectra of the MT2 probe in response to target RNA. The $10 \mu M$ probe RNA was

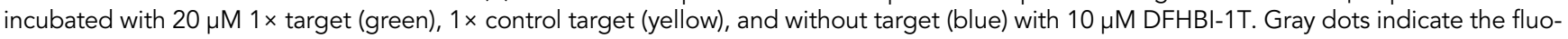
rescence spectrum of $10 \mu \mathrm{M}$ DFHBI-1T only.

To check its sequence selectivity, the changes in fluorescence of the BRD probe incubated with target RNA, control target RNA, and no target RNA were investigated. To prepare the control target RNA, the sequence of target RNA was completely randomized (Supplemental Table S2). As shown in Figure 3F, the control target RNA did not enhance fluorescence of the BRD probe, whereas the target RNA remarkably enhanced fluorescence. Furthermore, the BRD probe complexed with the target RNA exhibited a $507 \mathrm{~nm}$ peak, similar to Broccoli (Filonov et al. 2014). Thus, the BRD probe can selectively detect target RNAs by enhancing its fluorescence.

To determine whether the BRD probe is capable of targeting internal sequences of a long $m R N A$, we prepared mRNA encoding EGFP (Supplemental Table S2; Supplemental Note). In the presence of $2.5 \mu \mathrm{M}$ EGFP mRNA, the BRD probe showed about fourfold fluorescence enhancement of DFHBI-1T (Supplemental Fig. S2). This re- sult indicates that the BRD probe is able to respond to internal sequences of a long mRNA.

\section{Fluorescence characterization of BRD probe possessing MT2 three-way junction}

To explore the universal applicability of the BRD probe for detecting various RNA sequences, we designed four distinct BRD probes targeting different RNA sequences derived from endogenous mRNAs in humans: Actin $\beta$ (ACTB), Cyclin A2 (CCNA2), Cortactin (CTTN), and KRAS Proto-Oncogene, GTPase (KRAS) (Supplemental Table S2). The detailed procedure of probe design is shown in Materials and Methods. ACTB is one of the most famous and well-characterized genes whose mRNA is specifically transported to the cytoskeleton (Fusco et al. 2003; Yoshimura et al. 2012). Therefore, the mRNA of ACTB is usually used as a model of RNA detection. In addition, 
RNA sequences derived from CCNA2, CTTN, and KRAS were chosen to demonstrate their use in disease diagnosis, since they are known biomarkers of breast, squamous, and colorectal cancer, respectively (Lièvre et al. 2006; Hofman et al. 2008; Uhlen et al. 2010). To prepare the target RNAs, 52-nt internal sequences of these endogenous mRNAs were in vitro transcribed. The probe RNA and target RNA sequences are shown in Figure 4A and Supplemental Table S2. The three-way junction of the BRD probe against $A C T B$ is also illustrated in the left of Figure $4 B$. As shown in Figure 4C-F, each BRD probe showed 4.3-, 33-, 11-, and 48-fold fluorescence enhancement in response to the target RNAs, ACTB, CCNA2, CTTN, and KRAS, respectively. Among these probes, ACTB exhibited the highest background signal, suggesting that its target recognition sequence partially stabilizes the P1 stem and slightly enhances fluorescence. In contrast, the other probes showed lower background fluorescence. Considering these findings, optimization of the target recognition sequence is important for decreasing background signal. To assess the detection sensitivity of the BRD probe, a target RNA titration experiment was performed. The BRD probe showed increased fluorescence intensity with increasing concentrations of target RNA between $80 \mathrm{nM}$ and $20 \mu \mathrm{M}$ and could significantly detect small amounts of target RNA_as low as 160 nM (Fig. 4G). Next, we examined whether the BRD probe can distinguish its target RNA in a complex RNA mixture, such as total RNA extracted from yeast. As shown in Supplemental Figure S3, the BRD probe showed fluorescence enhancement with its target RNA even in the presence of $0.83 \mu \mathrm{g} / \mu \mathrm{L}$ yeast total RNA (the mass concentration of $20 \mu \mathrm{M}$ target RNA is $0.33 \mu \mathrm{g} /$ $\mu \mathrm{L})$. Moreover, this fluorescence response was comparable to that in the absence of yeast total RNA. These results reveal that the BRD probe can specifically enhance fluorescence intensity in response to its target RNA even in a complex mixture of RNA and that $160 \mathrm{nM}$ is the minimum detectable concentration of target RNA that can be significantly detected. Moreover, the BRD probe can be used to detect various endogenous RNA sequences.

\section{Construction and fluorescence characterization of BSRD probe with MT2 three-way junction}

We applied the MT2 three-way junction to Baby Spinach, which is another stem-loop aptamer that binds to DFHBI1T. The constructed BSRD probe is shown in Figure 4A and the middle of Figure 4B. The BSRD probe showed 3.0-, 62-, 11-, and 140-fold fluorescence enhancement in response to the target RNAs, ACTB, CCNA2, CTTN, and KRAS, respectively (Fig. 4C-F). Next, a target RNA titration experiment was performed to check the detection sensitivity of the BSRD probe. Surprisingly, the BSRD probe exhibited increased fluorescence intensity with increasing concentrations of target RNA from $2 \mathrm{nM}$ to $20 \mu \mathrm{M}$ and could significantly detect very small amounts of target RNA that were as low as $5 \mathrm{nM}$ (Fig. $4 \mathrm{H})$. This response behavior was superior to that of the BRD probe. Moreover, the BSRD probe is also able to respond to internal sequences of a long mRNA (Supplemental Fig. S2) and detect its target RNA in a complex RNA mixture (Supplemental Fig. S3). These results suggest that the MT2 three-way junction with the 14-nt target recognition sequences might be used as a scaffold for producing structure-switching aptamers, which enables programmable RNA detection. Interestingly, the previously reported BHQ-binding RNA aptamer system also has an MT2-like three-way junction (Sato et al. 2015). Considering these findings, there are some possibilities that the MT2 threeway junction can be applied to other stem-loop fluorescent aptamers, including Mango, red Broccoli, and orange Broccoli (Dolgosheina et al. 2014; Song et al. 2017) to construct various, colored RNA detection probes. Furthermore, combined with the protein-binding stem-loop aptamers, including MS2 aptamer and PP7 aptamer (Peabody 1993; Lim et al. 2001), the MT2 three-way junction might enable programmable editing, localization control, and degradation of target RNAs.

Ong et al. (2017) recently reported an RNA detection probe based on mini Spinach, which is a variant of Spinach and called it a "turn-on" probe. The "turn-on" probe also enables for programmable RNA detection with up to a 10-fold fluorescence enhancement in the presence of the target RNA. As shown in Figure $4 A$ and the right panel of Figure 4B, the "turn-on" probe was constructed from a truncated mutant of mini Spinach fused to 11-nt target recognition sequences on each end without any bridges between them or a single-strand structure at the center of the target RNA. To compare the BRD, BSRD, and "turnon" probes with regard to their fluorescent responsiveness, the "turn-on" probes against ACTB, CCNA2, CTTN, and KRAS RNA were designed (Fig. 4A, the right of Fig. 4B; Supplemental Table S2). As shown in Figure 4C-F, compared to the "turn-on" probe, the BRD and BSRD probes showed superior fluorescence enhancement in response to their target RNA sequences, which were derived from human mRNAs. We then tested four more target RNA sequences derived from mRNAs dnaK, dnaJ, adK, and mreB found in $E$. coli, which had been used to characterize the "turn-on" probe in the previous study (Ong et al. 2017). As shown in Supplemental Figure S4, the BRD and BSRD probes also showed superior fluorescence enhancement in response to three out of the four target RNAs compared to the "turn-on" probe. To determine whether the superior responses of the BRD and BSRD probes are owing to a characteristic of DFHBI-1T, the fluorescence responses of the $B R D, B S R D$, and "turn-on" probes against CTTN RNA were also evaluated using DFHBI. As shown in Supplemental Figure S5, a similar trend was observed even when DFHBI was used as a fluorophore instead of DFHBI-1T. 
A

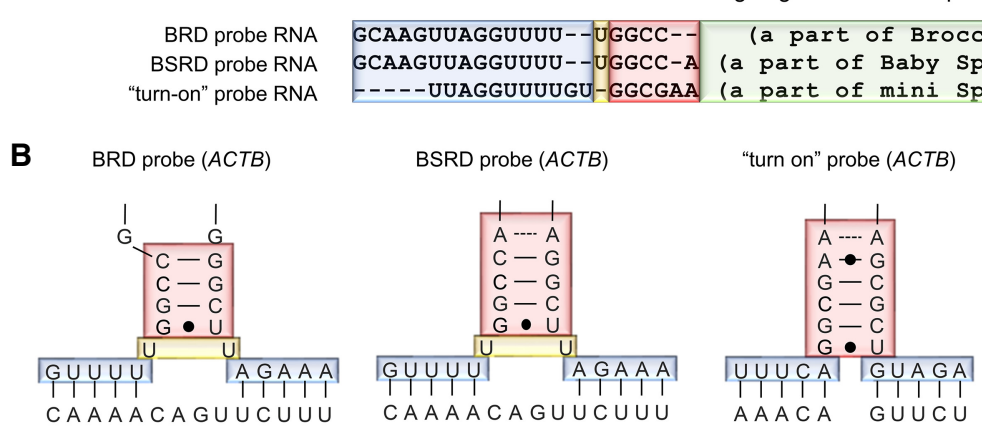

C

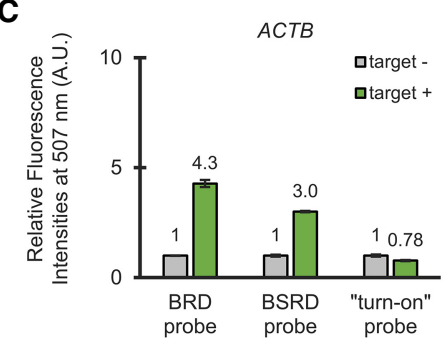

E

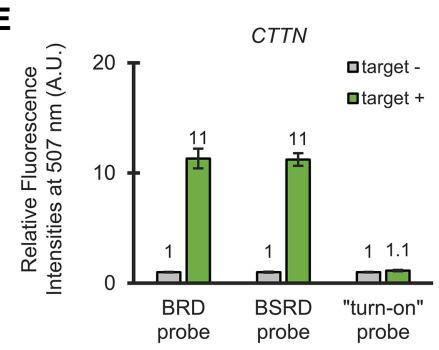

Probe design against $A C T B$ sequence

\begin{tabular}{l|l|l} 
ch) & - A-GCUU--AGAAAGGGUGUAAC \\
Ch) & AGCGCC-CAAGAAAGGGU----
\end{tabular}

G $\quad$ BRD probe (KRAS)
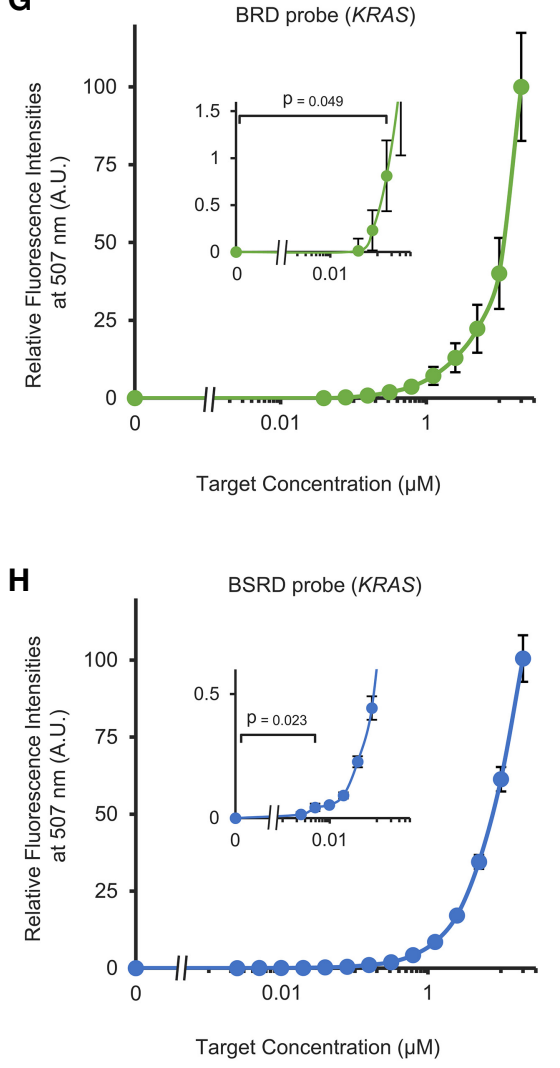

FIGURE 4. Characterization of the BRD and BSRD probes. (A) Sequence alignment of the BRD, BSRD, and "turn-on" probes against the ACTB sequence. (B) Secondary structure illustrations of the three-way junctions of the BRD, BSRD, and "turn-on" probe against the ACTB sequence. A part of the aptamer, the P1 stems, the $U$ mononucleotide bridges, and the target RNA recognition sequences are highlighted in green, red, yellow, and blue, respectively. (C-F) Fluorescence responses of BRD, BSRD, and "turn-on" probes against four distinct endogenous sequences: (C) ACTB, (D) CCNA2, (E) CTTN, and (F) KRAS of human. The $10 \mu \mathrm{M}$ probe RNA was incubated in the absence (gray) or presence (green) of $20 \mu \mathrm{M}$ target RNA with $10 \mu \mathrm{M}$ DFHBI-1T. The fluorescence intensities were standardized to that of the probe in the absence of target RNA. Values shown are the means \pm SEs of $n=3$. $(G, H)$ Fluorescence titration curves of $(G)$ the BRD and $(H) B S R D$ probes against various concentrations of KRAS sequence. The $10 \mu \mathrm{M}$ probe RNA was incubated with $10 \mu \mathrm{M}$ DFHBI-1T and different concentrations of target RNA. Values shown are the means \pm SEs of $n=3$ experiments. $P$-values were calculated using Independent-Samples Student's $t$-test.

These results indicate that, among fluorescent aptamerbased RNA detection probes, the BRD and BSRD are highly sensitive and universally applicable for detecting various RNAs (Table 1).

\section{Conclusion}

In the present study, we structurally characterized the Broccoli-DFHBI-1T complex, which is one of the brightest fluorescent aptamers. The CD spectra and UV melting measurements revealed that Broccoli was composed of a G-quadruplex and a double-strand stem structure, respectively. From these results, we predicted its structures as having a G-quadruplex-based DFHBI-1T recognition region sandwiched between two stems. Based on this 3D structure and the prediction of thermodynamic stability, we developed the BRD probe. The MT2 three-way junction of the BRD probe-target RNA complex has structural flexibility around the mononucleotide bridges between the P1 stem and the target recognition sequences in keeping with the thermodynamic stability of the P1 stem. Therefore, it efficiently induces refolding of Broccoli in the presence of the target RNA and enables for programmable RNA detection with a high signal-to-noise ratio and sensitivity. Moreover, the MT2 three-way junction can be used for probe construction using Baby Spinach. The obtained BSRD probe also enables for programmable RNA detection with better responses than the BRD probe. Since the BRD and BSRD probes consist of only an unmodified, short RNA and the cell-permeable, small molecule DFHBI-1T, 
TABLE 1. Fluorescent properties of BRD, BSRD, and "turn-on" probes

\begin{tabular}{|c|c|c|c|}
\hline & $\begin{array}{l}\text { Maximum } \\
\text { enhancement } \\
\text { ratio (fold) }\end{array}$ & $\begin{array}{l}\text { Applicable } \\
\text { target } \\
\text { sequence } \\
(\%)^{a}\end{array}$ & $\begin{array}{l}\text { Minimum } \\
\text { detectable } \\
\text { concentration } \\
(\mathrm{nM})^{\mathrm{b}}\end{array}$ \\
\hline BRD probe & 48 & 88 & 160 \\
\hline BSRD probe & 140 & 100 & 5 \\
\hline "Turn-on" probe & 12 & 50 & - \\
\hline \multicolumn{4}{|c|}{$\begin{array}{l}\text { "When probe exhibited more than twofold fluorescence enhancement in } \\
\text { the presence of } 20 \mu \mathrm{M} \text { target RNA, it was defined as "detected" in this } \\
\text { study. } \\
\text { bThe minimum concentration of target RNA which could be significantly } \\
\text { detected using the said probe. }\end{array}$} \\
\hline
\end{tabular}

the probe for a given target RNA can be easily designed for both in vitro and in vivo applications. Combined with the previously reported BHQ-binding RNA aptamer system (Sato et al. 2015), the BRD and BSRD probes would enable for real-time multicolor imaging of endogenous RNAs in live cells.

The in vitro detection of endogenous RNAs is important for assessing cellular conditions and diagnosing diseases. In the present study, we demonstrated the detection of several cancer biomarkers using the BRD and BSRD probes by only changing the target recognition sequences. This programmable RNA detection method will accelerate therapeutic diagnosis for a wide variety of cancers, virus-, and bacteria-related diseases. More recently, an RNA imaging method designed with two split fragments of Broccoli has been reported (Wang et al. 2018). While the minimum detectable concentration of the splitBroccoli system is about $50-100 \mathrm{nM}$, it only exhibits up to an eightfold fluorescence enhancement in the presence of its target RNA. Karunanayake Mudiyanselage et al. (2018) also reported a genetically encoded RNA-based catalytic hairpin assembly circuit (CHARGE) method for sensitive RNA detection using split Broccoli. In this system, one target RNA catalytically activates tens-to-hundreds of Broccoli fluorescence. Thus, CHARGE is able to detect as low as 2.5 nM RNA target, whereas this circuit requires two components. As we showed in the present study, the BRD and BSRD probes exhibit up to 48- and 140-fold fluorescence enhancement with one component in the presence of the target RNA, respectively. Furthermore, the BSRD probe can detect a very small amount of target RNA—as low as $5 \mathrm{nM}$-while the minimum detectable concentration of the BRD probe is $160 \mathrm{nM}$. These results reveal that the BRD and BSRD probes possess high signal-tonoise ratios, and the BSRD probe possesses the highest level of sensitivity among all RNA detection probes based on fluorescent aptamers. However, we think that our probes are not able to discriminate a slight sequence difference such as single nucleotide mutation or detect extremely low-abundant RNAs. For example, let us suppose that we detect a low copy mRNA such as one molecule per cell. Assuming that the cell volume is $2500 \mu^{3}$ and probes can be expressed at 1000 molecules per cell, the dissociation constant between the target and probe should be $\sim 670 \mathrm{pM}$ to form a complex. Moreover, the probe requires 1000 -folds or more fluorescence enhancement when it is assumed that the total fluorescence intensity needs to be doubled for detection. Ongoing work will focus on improving the specificity and fluorescence enhancement ratio to achieve the detection of single nucleotide mutation and extremely low-abundant RNAs.

\section{MATERIALS AND METHODS}

\section{D structure prediction of Broccoli}

The sequence alignment of Broccoli and Baby Spinach RNA was performed using A plasmid Editor (ApE) (Davis 2018). The predicted 3D structure of Broccoli was illustrated using HyperChem Release 7.51 (Hypercube Inc.) and MacroModel v10.4 (Schrödinger, LLC) based on the Spinach RNA aptamer crystal structure (PDB ID: 4TS2) (Warner et al. 2014). First, using HyperChem Release 7.51 , the structure of the DFHBI-1T recognition region was extracted (PDB ID: 4TS2). According to the sequence alignment result, A27 and $U 62$ were changed to $U$ and $C$, respectively. The P1 stem and P2 stem-cap structure of Broccoli were created using the Nucleic Acids tool and energy minimized in the presence of water using the Geometry Optimization tool with the Polak-Ribiere algorithm. Then, the P1 stem and P2 stem-cap structures were fused to the mutated DFHBI-1T recognition region. Finally, using MacroModel, the overall structure of Broccoli was energy minimized with the PRCG algorithm (convergence threshold, 0.05 mol $^{-1} \AA^{-1}$ ) using the AMBER* force field and GB/SA continuum water model.

\section{Probe design}

RNA sequences of BRD and BSRD probes were designed with the following requirements. (i) The GC content of target recognition sequences is between $25 \%$ and $60 \%$. (ii) There are no runs of five or more of one base, or dinucleotide repeats in target recognition sequences. (iii) There are no similar or complementary sequences to Broccoli or Baby Spinach sequences in target recognition sequences. (iv) There are no highly similar sequences to the selected target RNA sequences in human RNAs. Nucleotide BLAST was used for the sequence alignment.

\section{Preparation of RNAs}

The dsDNA templates for Broccoli mutant, Broccoli probe, "turnon" probe, and short target RNA synthesis were prepared by Klenow reaction of individual ssDNA containing the T7 promoter sequence (Supplemental Table S1) using the Klenow fragment (cat. no. 2140A; Takara Bio Inc.) and T7 primer. The dsDNA templates for synthesizing the BSRD probe RNA were prepared by 
PCR using the GoTaq Green Master Mix (cat. no. M7122; Promega Corporation) and DNA oligonucleotides (Supplemental Table 1). The dsDNA template for synthesizing EGFP mRNA were PCR amplified using the pcDNA3.1 EGFP plasmid as a template, with primers (5'-TAA TAC GAC TCA CTA TAG GGA-3' and 5'-CCA TAG AGC CCA CCG CAT CC-3'). RNAs were transcribed from these dsDNA templates by in vitro transcription reactions at $37^{\circ}$ C for 150 min using the ScriptMAX Thermo T7 Transcription Kit (cat. no. TSK-101; Toyobo Co., Ltd.), and purified using Micro Bio-Spin 6 columns (cat. no. 7326221; Bio-Rad Laboratories, Inc.) to remove unincorporated rNTPs. Denaturing urea polyacrylamide gel electrophoresis (Urea-PAGE) was performed using $5 \mathrm{M}$ urea and $10 \%$ polyacrylamide gels to ensure that transcribed RNAs were of the proper length.

\section{Circular dichroism (CD) measurements}

$\mathrm{CD}$ spectra were measured in Tris buffer (10 mM Tris- $\mathrm{HCl}[\mathrm{pH} 7.5]$, $5 \mathrm{mM} \mathrm{MgCl}_{2}$, and $10 \mu \mathrm{M}$ DFHBI-1T) (Fig. 2B) or in HEPES buffer (40 mM HEPES [pH 7.4], $100 \mathrm{mM} \mathrm{KCl}, 1 \mathrm{mM} \mathrm{MgCl}$, and $10 \mu \mathrm{M}$ DFHBI-1T) (Supplemental Fig. S1) using $100 \mu \mathrm{L}$ of sample. The concentration of RNA was $5 \mu \mathrm{M}$. DFHBI-1T (cat. no. 410-1 mg) was purchased from Lucerna, Inc. DFHBI-1T was dissolved in water or DMSO for the preparation of Tris and HEPES buffer, respectively. Samples were incubated at $37^{\circ} \mathrm{C}$ for $30 \mathrm{~min}$. Measurements were performed using a CD Spectrometer (JASCO-1500, JASCO Corporation). Five scans from 200 to $320 \mathrm{~nm}$ at $0.5 \mathrm{~nm}$ intervals were accumulated with a scan rate of $100 \mathrm{~nm} \mathrm{~min}^{-1}$ and averaged (path length $=1 \mathrm{~mm}$ ).

\section{UV melting measurements}

UV absorbance was measured in a buffer (40 mM HEPES pH 7.4, $100 \mathrm{mM} \mathrm{KCl}, 1 \mathrm{mM} \mathrm{MgCl} 2,10 \mu \mathrm{M}$ DFHBI-1T) at the volume of 100 $\mu \mathrm{L}$. The concentration of RNA was $5 \mu \mathrm{M}$. Samples were incubated at $37^{\circ} \mathrm{C}$ for $30 \mathrm{~min}$ and were transferred to a $1 \mathrm{~cm}$ path length quartz cuvette and then covered with a layer of liquid paraffin. UV melting curves were collected using a UV Spectrophotometer (UV-2450, Shimadzu Corporation) measuring the absorbance at $260 \mathrm{~nm}$. Melting measurements were performed from $4^{\circ} \mathrm{C}$ to $110^{\circ} \mathrm{C}$ with a $0.5^{\circ} \mathrm{C} / \mathrm{min}$ heating rate and constant flow of dry nitrogen to prevent dew.

\section{Fluorescence measurements}

The fluorescent properties of RNA aptamers and probes were measured in a buffer (40 mM HEPES pH 7.4, $100 \mathrm{mM} \mathrm{KCl,} 1$ $\mathrm{mM} \mathrm{MgCl} 2,10 \mu \mathrm{M} \mathrm{DFHBI}-1 \mathrm{~T}$, or $10 \mu \mathrm{M} \mathrm{DFHBI}$ ) at a total volume of $5 \mu \mathrm{L}$. DFHBI (cat. no. SML1627-25MG) was purchased from Merck KGaA. For the fluorescence measurements of truncated Broccoli mutants, the concentration of RNA was $1 \mu \mathrm{M}$. For characterization of the RNA detection probe using short target RNA, probe RNA $(10 \mu \mathrm{M})$ was hybridized with $2 \times$ target RNA $(10 \mu \mathrm{M})$ or $1 \times$ target RNA $(20 \mu \mathrm{M})$. For characterization of the RNA detection probe using full-length mRNA, probe RNA $(10 \mu \mathrm{M})$ was hybridized with EGFP mRNA $(2.5 \mu \mathrm{M})$. For characterization of the specificity of the RNA detection probe, probe RNA (10 $\mu \mathrm{M})$ was hybridized with target RNA $(20 \mu \mathrm{M}, 0.33 \mu \mathrm{g} / \mu \mathrm{L})$ in the absence or presence of $0.83 \mu \mathrm{g} / \mu \mathrm{L}$ yeast RNA (cat. no. AM7120G; Thermo Fisher Scientific). For the target RNA titration experiment, probe RNA $(10 \mu \mathrm{M})$ was hybridized with different concentrations of target RNA. Samples were incubated at $37^{\circ} \mathrm{C}$ for $30 \mathrm{~min}$, and the fluorescence spectra of the samples were measured using an excitation wavelength of $472 \mathrm{~nm}$ and an emission range from 490 to $700 \mathrm{~nm}$ using a spectrofluorometer (FP-8300, JASCO Corporation).

\section{Statistical analysis}

All measurements are presented as the mean \pm standard error (SE). Statistical significance was evaluated using the Independent-Samples Student's t-test.

\section{SUPPLEMENTAL MATERIAL}

Supplemental material is available for this article.

\section{ACKNOWLEDGMENTS}

We thank Y. Nihongaki and M. Sato for their technical assistance. This work was supported by the Japan Society for the Promotion of Science (JSPS) KAKENHI (grant numbers 24350037, 15K13721, 16H04169, and 16K16398), the A-STEP program (AS262ZO29 08P), the Casio Science Promotion Foundation, the Moritani Scholarship Foundation, the Nagase Science Technology Foundation, and the JSPS Research Fellowships for Young Scientists (grant number 15J08446).

Received October 2, 2018; accepted February 6, 2019.

\section{REFERENCES}

Ageely EA, Kartje ZJ, Rohilla KJ, Barkau CL, Gagnon KT. 2016. Quadruplex-flanking stem structures modulate the stability and metal ion preferences of RNA mimics of GFP. ACS Chem Biol 11: 2398-2406. doi:10.1021/acschembio.6b00047

Alberts B, Johnson A, Raff LJ, Roberts M, Walter PK. 2008. Molecular biology of the cell. Garland Science, New York.

Autour A, Westhof E, Ryckelynck M. 2016. iSpinach: a fluorogenic RNA aptamer optimized for in vitro applications. Nucleic Acids Res 44: 2491-2500. doi:10.1093/nar/gkw083

Chiu YL, Ali A, Chu CY, Cao H, Rana TM. 2004. Visualizing a correlation between siRNA localization, cellular uptake, and RNAi in living cells. Chem Biol 11: 1165-1175. doi:10.1016/j.chembiol.2004.06 .006

DasGupta S, Shelke SA, Li N, Piccirilli JA, Guo L, Wang H, Liang H. 2015. Spinach RNA aptamer detects lead(II) with high selectivity. Chem Commun 51: 9034-9037. doi:10.1039/C5CC01526J

Davis MW. 2018. ApE—a Plasmid Editor. http://www.biology.utah .edu/jorgensen/wayned/ape

Dolgosheina EV, Jeng SCY, Panchapakesan SSS, Cojocaru R, Chen PSK, Wilson PD, Hawkins N, Wiggins PA, Unrau PJ. 2014. RNA mango aptamer-fluorophore: a bright, high-affinity complex for RNA labeling and tracking. ACS Chem Biol 9: 2412-2420. doi:10.1021/cb500499x

Filonov GS, Moon JD, Svensen N, Jaffrey SR. 2014. Broccoli: rapid selection of an RNA mimic of green fluorescent protein by fluorescence-based selection and directed evolution. J Am Chem Soc 136: 16299-16308. doi:10.1021/ja508478x 
Freeman WM, Walker SJ, Vrana KE. 1999. Quantitative RT-PCR: pitfalls and potential. Biotechniques 26: 112-125. doi:10.2144/ 99261 rv01

Fusco D, Accornero N, Lavoie B, Shenoy SM, Blanchard JM, Singer RH, Bertrand E. 2003. Single mRNA molecules demonstrate probabilistic movement in living mammalian cells. Curr Biol 13: 161-167. doi:10.1016/S0960-9822(02)01436-7

Higuchi R, Dollinger G, Sean Walsh P, Griffith R. 1992. Simultaneous amplification and detection of specific DNA sequences. Nat Biotechnol 10: 413-417. doi:10.1038/nbt0492-413

Hofman P, Butori C, Havet K, Hofman V, Selva E, Guevara N, Santini J, Van Obberghen-Schilling E. 2008. Prognostic significance of cortactin levels in head and neck squamous cell carcinoma: comparison with epidermal growth factor receptor status. Br J Cancer 98: 956-964. doi:10.1038/sj.bjc.6604245

Huang $H$, Suslov NB, Li NS, Shelke SA, Evans ME, Koldobskaya Y, Rice PA, Piccirilli JA. 2014. A G-quadruplex-containing RNA activates fluorescence in a GFP-like fluorophore. Nat Chem Biol 10: 686-691. doi:10.1038/nchembio.1561

Karunanayake Mudiyanselage APKK, Yu Q, Leon-Duque MA, Zhao B, Wu R, You M. 2018. Genetically encoded catalytic hairpin assembly for sensitive RNA imaging in live cells. J Am Chem Soc 140: 8739-8745. doi:10.1021/jacs.8b03956

Ketterer S, Fuchs D, Weber W, Meier M. 2015. Systematic reconstruction of binding and stability landscapes of the fluorogenic aptamer spinach. Nucleic Acids Res 43: 9564-9572. doi:10.1093/nar/ gkv944

Li J, Tan S, Kooger R, Zhang C, Zhang Y. 2014. MicroRNAs as novel biological targets for detection and regulation. Chem Soc Rev 43: 506-517. doi:10.1039/C3CS60312A

Lièvre $A$, Bachet JB, Le Corre $D$, Boige V, Landi B, Emile JF, Côté JF, Tomasic G, Penna C, Ducreux M, et al. 2006. KRAS mutation status is predictive of response to cetuximab therapy in colorectal cancer. Cancer Res 66: 3992-3995. doi:10.1158/0008-5472.CAN06-0191

Lim F, Downey TP, Peabody DS. 2001. Translational repression and specific RNA binding by the coat protein of the Pseudomonas phage PP7. J Biol Chem 276: 22507-22513. doi:10.1074/jbc .M102411200

Mao X, Xu H, Zeng Q, Zeng L, Liu G. 2009. Molecular beacon-functionalized gold nanoparticles as probes in dry-reagent strip biosensor for DNA analysis. Chem Commun 125: 3065-3067. doi:10.1039/b822582f

Martin KC, Ephrussi A. 2009. mRNA localization: gene expression in the spatial dimension. Cell 136: 719-730. doi:10.1016/j.cell .2009.01.044

Okuda M, Fourmy D, Yoshizawa S. 2017. Use of baby spinach and broccoli for imaging of structured cellular RNAs. Nucleic Acids Res 45: 1404-1415. doi:10.1093/nar/gkx970

Ong WQ, Citron YR, Sekine S, Huang B. 2017. Live cell imaging of endogenous mRNA using RNA-based fluorescence "turn-on" probe. ACS Chem Biol 12: 200-205. doi:10.1021/acschembio $.6 \mathrm{~b} 00586$

Paige JS, Wu KY, Jaffrey SR. 2011. RNA mimics of green fluorescent protein. Science 333: 642-646. doi:10.1126/science.1207339

Peabody DS. 1993. The RNA binding site of bacteriophage MS2 coat protein. EMBO J 12: 595-600. doi:10.1002/j.1460-2075.1993 .tb05691.x

Sato SI, Watanabe M, Katsuda Y, Murata A, Wang DO, Uesugi M. 2015. Live-cell imaging of endogenous mRNAs with a small molecule. Angew Chemie Int Ed 54: 1855-1858. doi:10.1002/anie 201410339

Song W, Strack RL, Svensen N, Jaffrey SR. 2014. Plug-and-play fluorophores extend the spectral properties of Spinach. J Am Chem Soc 136: 1198-1201. doi:10.1021/ja410819x

Song W, Filonov GS, Kim H, Hirsch M, Li X, Moon JD, Jaffrey SR. 2017. Imaging RNA polymerase III transcription using a photostable RNA-fluorophore complex. Nat Chem Biol 13: 1187-1194. doi:10.1038/nchembio. 2477

Svensen N, Jaffrey SR. 2016. Fluorescent RNA aptamers as a tool to study RNA-modifying enzymes. Cell Chem Biol 23: 415-425. doi:10.1016/j.chembiol.2015.11.018

Tyagi S, Kramer FR. 1996. Molecular beacons: probes that fluoresce upon hybridization. Nat Biotechnol 14: 303-308. doi:10.1038/ nbt0396-303

Uhlen M, Oksvold P, Fagerberg L, Lundberg E, Jonasson K, Forsberg M, Zwahlen M, Kampf C, Wester K, Hober S, et al. 2010. Towards a knowledge-based human protein atlas. Nat Biotechnol 28: 1248-1250. doi:10.1038/nbt1210-1248

Varani G, McClain WH. 2000. The G.U wobble base pair: a fundamental building block of RNA structure crucial to RNA function in diverse biological systems. EMBO Rep 1: 18-23. doi:10.1093/ embo-reports/kvd001

Wang Z, Luo Y, Xie X, Hu X, Song H, Zhao Y, Shi J, Wang L, Glinsky G, Chen $N$, et al. 2018. In situ spatial complementation of aptamermediated recognition enables live-cell imaging of native RNA transcripts in real time. Angew Chemie Int Ed 57: 972-976. doi:10.1002/anie.201707795

Warner KD, Chen MC, Song W, Strack RL, Thorn A, Jaffrey SR, FerréD'Amaré AR. 2014. Structural basis for activity of highly efficient RNA mimics of green fluorescent protein. Nat Struct Mol Biol 21: 658-663. doi:10.1038/nsmb.2865

Xia Y, Zhang R, Wang Z, Tian J, Chen X. 2017. Recent advances in high-performance fluorescent and bioluminescent RNA imaging probes. Chem Soc Rev 46: 2824-2843. doi:10.1039/c6cs00675b

Yeh HY, Yates MV, Mulchandani A, Chen W. 2010. Molecular beaconquantum dot-Au nanoparticle hybrid nanoprobes for visualizing virus replication in living cells. Chem Commun 46: 3914-3916. doi:10.1039/c001553a

Yoshimura H, Inaguma A, Yamada T, Ozawa T. 2012. Fluorescent probes for imaging endogenous $\beta$-actin mRNA in living cells using fluorescent protein-tagged pumilio. ACS Chem Biol 7: 999-1005. doi:10.1021/cb200474a 

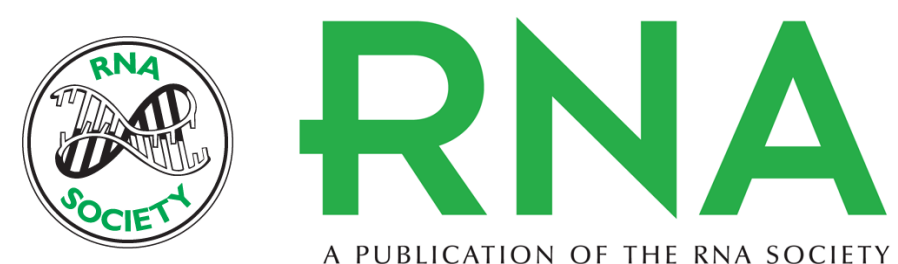

A PUBLICATION OF THE RNA SOCIETY

\section{Programmable RNA detection with a fluorescent RNA aptamer using optimized three-way junction formation}

Yuichi Furuhata, Mizuki Kobayashi, Ryo Maruyama, et al.

RNA 2019 25: 590-599 originally published online February 11, 2019

Access the most recent version at doi:10.1261/rna.069062.118

\section{Supplemental http://rnajournal.cshlp.org/content/suppl/2019/02/11/rna.069062.118.DC1 Material}

References This article cites 34 articles, 3 of which can be accessed free at: http://rnajournal.cshlp.org/content/25/5/590.full.html\#ref-list-1

Creative This article is distributed exclusively by the RNA Society for the first 12 months after the Commons License full-issue publication date (see http://rnajournal.cshlp.org/site/misc/terms.xhtml). After 12 months, it is available under a Creative Commons License (Attribution-NonCommercial 4.0 International), as described at http://creativecommons.org/licenses/by-nc/4.0/.
Email Alerting Receive free email alerts when new articles cite this article - sign up in the box at the Service top right corner of the article or click here.

To subscribe to $R N A$ go to:

http://rnajournal.cshlp.org/subscriptions 